\title{
Flexible coordination and information sharing for virtual enterprises in PRODNET
}

\author{
L. M. Camarinha-Matos ${ }^{1)}$, H. Afsarmanesh ${ }^{2)}$ \\ 1) New University of Lisbon, Quinta da Torre, 2825 Monte \\ Caparica, Portugal \\ Tel: +351-1-2948517 \\ Fax: +351-1-2941253 \\ e-mail:cam@uninova.pt \\ ${ }^{2)}$ University of Amsterdam, Kruislaan 403, $1098 \mathrm{SJ}$ \\ Amsterdam, The Netherlands \\ e-mail: hamideh@wins.uva.nl
}

\begin{abstract}
Flexible coordination and configuration is a major aspect in the development of open support infrastructures for Industrial Virtual Enterprises, particularly in the context of small and medium size enterprises. The steps involved in the creation and configuration of an infrastructure to support the life cycle of a virtual enterprise are introduced. The specific approach of hierarchical coordination infrastructure, being partly developed and applied to the Esprit PRODNET II project is presented. The coordination / information-sharing kernel is introduced as the composition of a hierarchical (workflow-like) activity management approach with a distributed / federated database management system.
\end{abstract}

Keywords

Virtual enterprises, coordination, federated information management.

\section{INTRODUCTION}

Cooperation is a key underlying aspect in the Virtual Enterprise (VE) paradigm as manufacturing processes are not carried on by a single enterprise anymore. In order to reach world class in open markets, companies feel the need to focus on their core competencies and joint efforts with others to fulfill the requirements of the new products / services demanded by the market. A VE is a temporary consortium 
of autonomous enterprises that decide to cooperate in order to achieve a common goal (i.e. to jointly respond to a business opportunity). This cooperation involves, in general, sharing information and other resources, communication, ,producer / consumer" relationships (information or product-related), and collaborative activities.

Flexible coordination and configuration is a major aspect in the development of open support infrastructures for Industrial Virtual Enterprises. The coordination issues in VEs in the context of Small and Medium sized Enterprises and the need for very flexible and easily configurable solutions are discussed in this paper. The specific approach of hierarchical coordination infrastructure, being partly developed and applied to the Esprit PRODNET II project is presented. The coordination / information-sharing kernel is introduced as the composition of a hierarchical (workflow-like) activity management approach with a distributed / federated database management system. It is explained how this kernel supports the coordination of enterprise activities in relation to its involvement in VEs, and some further implementation results are presented.

\section{ENTERPRISE FUNCTIONALITIES AND FLEXIBILITY}

A VE support infrastructure shall be highly configurable to allow easy definition and modification of desired behavior, be highly modular and incremental (open) in terms of offered services, support multiple activity coordination levels, and be based on a federated information management approach to provide all desired levels of information visibility and access rights required by involved enterprises. The Esprit PRODNET II aims at developing an open and highly flexible support infrastructure particularly suited to the needs of SMEs. In order to cope with the described requirements, the PRODNET approach includes a careful identification and modeling of all variable factors and the implementation of a clear separation between support services, information handling, and control mechanisms following a workflow management and federated database paradigm.

In particular, in the context of SMEs, a large diversity in the way companies do their business can be found. For instance, a company may use a strong humanbased control of the client orders processing, while others may prefer to rely on more automatic procedures implemented in the PPC (Production Planning and Control) or ERP (Enterprise Resources Planning) systems. In each company a number of support services may be available and the company has to handle a large number of cooperation-related events, but the way these events are treated and the services are activated is a local autonomous decision.

Activities carried out by a company are usually organized in "clusters" of interrelated activities called processes (business processes). Each process is designed in order to achieve a (partial) specific objective. When properly „orchestrated“, the combination of various processes will lead to the achievement of a global VE goal. The separation between services, the information, and the control part is a promising approach towards the implementation of flexible behavior. Therefore it 
is important that the behavior of each company regarding its participation in a VE can be explicitly configured through a plan and other general profile characteristics. One approach to represent the dynamic behavior is through the Workflow Process Definition Language following the general workflow reference architecture as proposed by the Workflow Management Coalition. At the same time, an approach to support the autonomy of each enterprise in sharing and exchange of its information with other enterprises can be properly supported through the federated information management system.

Therefore, one important requirement when designing an infrastructure to support virtual enterprises is to provide a high level of configurability such that the infrastructure can be adapted to a variety of operating scenarios. Namely, an effective PRODNET infrastructure must satisfy the requirements of the enterprise through the stages of installation and evolution within the VEs. Therefore, the two main stages include: (1) the initialization(/reinitialization) of the PRODNET infrastructure to the specificities of the enterprise environment and its business process behavior; and (2) the necessary configuration(/reconfiguration) of the infrastructure, to the VE specifications and regulations, for the enterprise's involvement within different virtual enterprises.

The main outcome of the initialization stage is the proper positioning and manifestation of the enterprise within the „PRODNET-compliant network of enterprises", and preparing it for getting involved in VEs. The outcome of the configuration stage however, is the proper setting of parameters for involvement of the enterprise within every VE, and support for the readjustment of these parameters, when necessary, during the VE's life cycle. For instance the reconfiguration necessary due to the "feedback" from the VE operation phase, when there is a need to select a new partner.

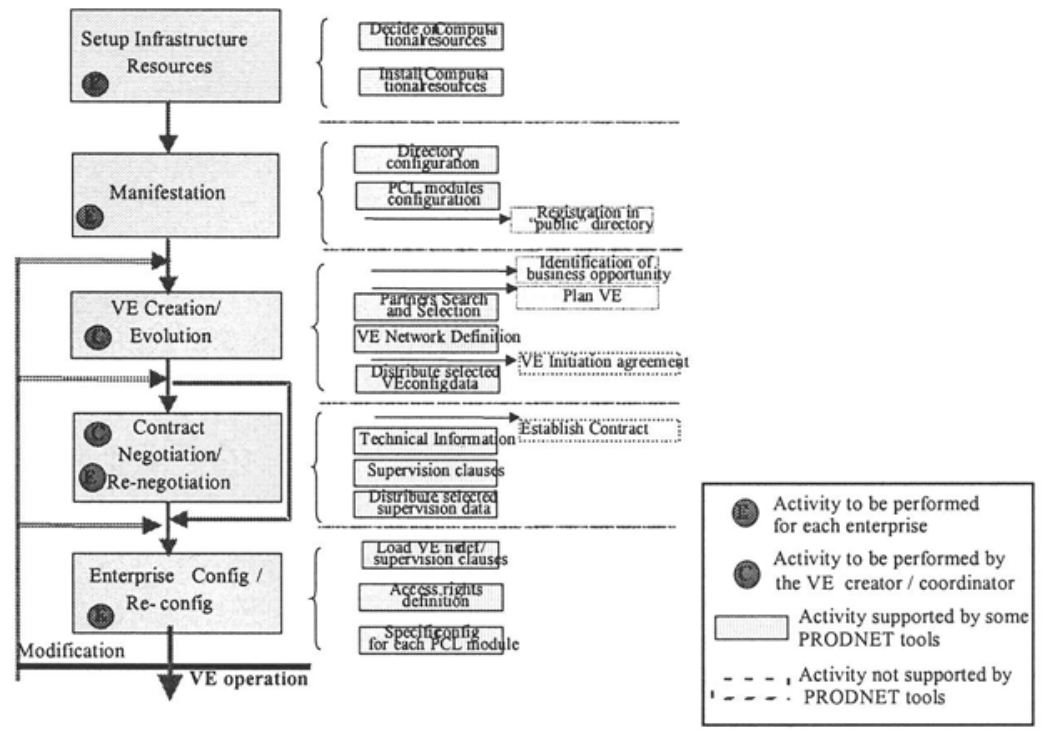

Figure 1 - Main steps in VE Initialization and configuration 
The main steps in the general process are illustrated in Figure 1. A brief description of every step follows:

- Setup of Infrastructure Resources: this step identifies and installs the computational resources for the enterprise.

- PRODNET Enterprise Manifestation: once the enterprise has properly installed its resources, as specified in the previous step, it needs to set up the PRODNET infrastructure components themselves, what is required to prepare the node for its future involvement in VEs. At this step the enterprise can "proclaim“ itself as an enterprise that is enabled with the PRODNET infrastructure.

- VE Creation/Evolution: when a business opportunity is identified, the proper VE partners are searched, identified and selected. Some preliminary agreements (,initiation agreement") will be reached, and then the VE topology and its partners' roles are defined and distributed among the VE-members. The same step needs to be followed, also in the case of a VE evolution (addition or substitution of partners).

- VE Contract Negotiation/Re-negotiation: once the topology of the VE and the role of its partners are defined, formal contracts need to be signed between the VE coordinator and individual partners. The contracts contain the rights and liabilities of enterprises towards the VE, and their agreement to the global VE rules and regulations. Both the technical production plans for the enterprises and the "Supervision Clauses" that define the rights of the VE coordinator to observe and monitor the progress of activities at the VE-member sites. Selected parts of supervision clause will then be distributed among the VE-members. Due to the VE evolution, new contracts may be negotiated among VE partners.

- Enterprise Configuration/Re-configuration: At this step, every VE partner loads the VE network definition, regulations, and supervision clauses, received from the VE coordinator. Based on these definitions, the enterprise will set the precise information access and visibility rights for every other VE-member and the various components of the PRODNET infrastructure will be tuned.

\section{HIERARCHICAL COORDINATION}

A challenge for a flexible infrastructure is the design of a hierarchical coordination mechanism. For instance, at least two distinct levels - VE level coordination and VE-member level coordination - should be supported by the infrastructure. Therefore, during the project, the initial PRODNET basic infrastructure evolved into a hierarchical architecture.

In order to support the above requirements a 3-layer hierarchical reference architecture is proposed by PRODNET, as represented by the Core Cooperation Layer (CCL), the Enterprise Cooperation Layer (ECL) and the PRODNET Cooperation Layer (PCL), defined hierarchically on top of each other, shown in figure 2. In this architecture, the so called ,management / coordination functionalities" are separated from the basic "cooperation functionalities“, as 
described bellow. Namely, every cooperation layer constitutes some management / coordination functionality as well as a lower level of cooperation layer.
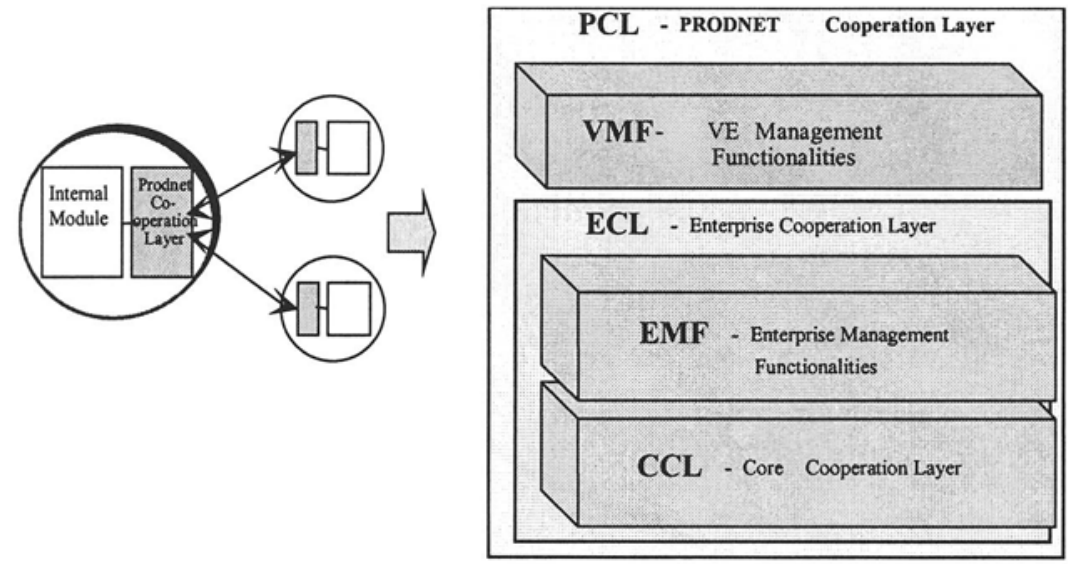

Figure 2 PRODNET Hierarchical Coordination

The role of the CCL includes: Safe communications, EDI and STEP based data exchange, definition of admission and visibility rights on both the public information (e.g. directory of services / products), and explicitly shared information (e.g. order status and current job status at different enterprises), etc. (Figure 3.a)
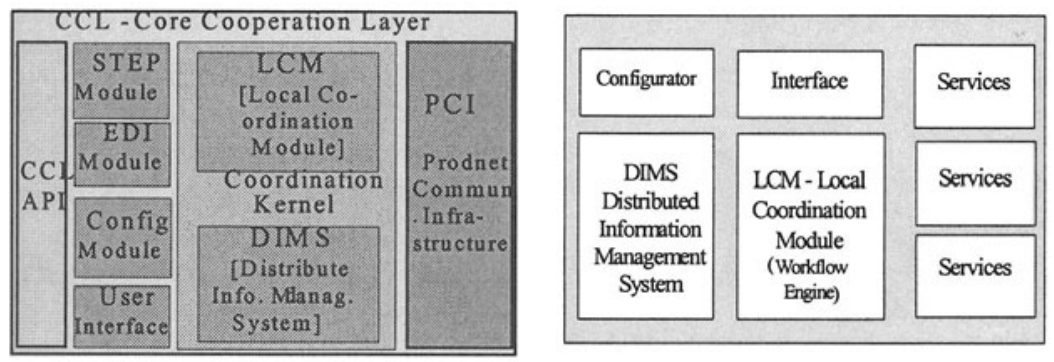

Figure 3 a) Core Cooperation Layer b) General structure of the EMF and VMF

The EMF module will provide a „re-engineered“ view of the enterprise's PPC/ERP system suited to operate in a VE environment. In PRODNET II some simple services to support contracts management and monitoring are implemented. Finally, VMF is responsible for the management of activities at the level of VE. For instance, functionalities like partners search and selection or distributed business processes management are located at this level. Each component of this architecture, namely CCL, EMF and VMF, has the same general structure as represented in (Figure 3.b). 
Enterprises involved in a VE, although competitors otherwise, need to share and exchange a part of their information in order to achieve the common goal of the virtual organization, and to support the VE functionalities as a single organization. Depending on the role played by different partners within the VE, different kinds of information need to be exchanged. For instance, partners involved in the supply chain require to exchange data about the product, while the VE coordinator enterprise needs to investigate the progress of work and job activities within the $\mathrm{VE}$ and at several individual partner sites. In a VE network however, on one hand, due to the competitiveness of pre-existing enterprises and their proprietary information, it is not realistic to assume a single global schema defining all the information that is visible and exchanged within the VE platform.

The support for enterprises autonomy can be provided through a distributed and federated cooperative architecture (the DIMS module in PRODNET), which is based on the definition of individual export schemas for enterprises within the VE, in order to support their authorization and information visibility rights. A natural consequence of this architecture is the support for security of shared data and provision of different access levels to shared data, based on the role(s) of each enterprise in the VE (Afsarmanesh, Camarinha-Matos, 1997).

Since different enterprises must have different visibility levels and access rights to other nodes' information, every node in the federation must decide what part of the information to make available to every other partner in a particular VE. The level of visibility and access that other partners have on the local PCL schema of a given node must be clearly determined. To accomplish this objective, every node can defend its autonomy and privacy by defining one detailed individual export schema on the PCL local schema, for every other node with which it shares information. Every export schema defines the corresponding access right of other node to this node's local data. For every class defined in the local schema, the individual export schema determines which instances (i.e. horizontal class partitioning) and which attributes (i.e. vertical class partitioning) of those instances will be made available to every other node. Through this mechanism, it is even possible to make the value of one specific attribute of a determined class instance, available to a given partner. On the other hand, it would be also possible to make all PCL information accessible for reading from another node.

The concept of individual export schema definition on the local schema for every external „user", is the basic idea. However, we have generalized this basic idea to the definition of a complete hierarchy of export schemas. The hierarchy of schemas allows the grouping and classification of common view characteristics, facilitating in this way the task of individual export schema definitions.

In the general case, two kinds of queries may arrive at a DIMS, an internal query (a query arriving from the PPC or other PCL modules in the node) or an external query (a query arriving from another node's DIMS). Furthermore, there are two kinds of internal queries that need to be handled. The query can be a local query which can be answered without acquiring any information from other nodes, 
or it can be a distributed query, which involves the retrieval of information from one or a specific set of nodes. In this way, the distributed query is based on the integrated schema, through which local and imported data is seamlessly accessed from any higher-level application interacting with the DIMS. For example, any query on the Partner_Info (at the PCL schema) involving other partners, must be sent to the corresponding nodes, where the query will be evaluated against the view definition (for this node) and the results (if any) will be sent back.

When a query arrives at the DIMS, it must first be determined if it is a local query or a distributed query. To make this distinction, the query must be parsed and analyzed in search of specifications of partners' ID corresponding to Other_Partners. Also, the query may contain a condition, representing some field specifications, that identify one or more VE partners (e.g. a "where" condition in a SQL query). In this case, a set of sub-queries will be deduced from the original query (one sub-query per partner), where the sub-queries will be sent, and finally the results will be merged.

\section{COORDINATION / INFORMATION-SHARING KERNEL}

There is a close relationship between the coordination module (workflow engine) and the DIMS in each of the 3 layers described in the hierarchical architecture above.

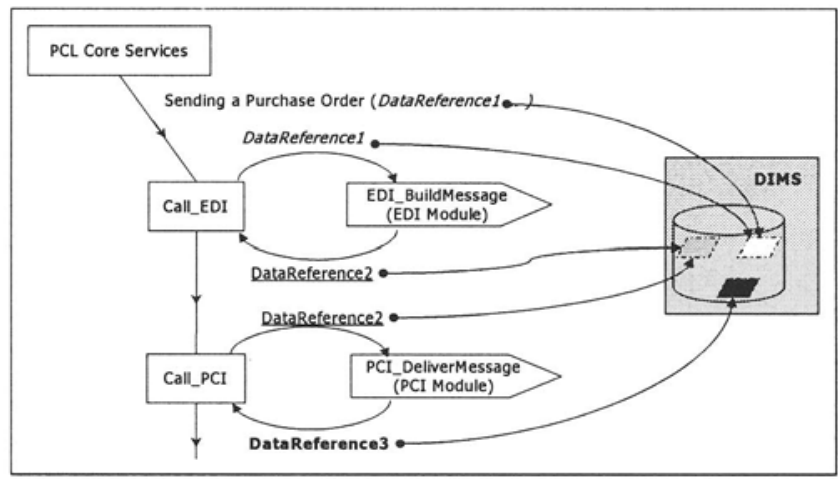

Figure 4 Interactions between workflow-based coordination and DIMS

The data associated to each activity being managed by a coordination module are stored in its corresponding DIMS (Figure 4), either as structured objects or as blobs. Workflow plans and the corresponding interactions with DIMS are configured for each type of event (sending out an order, for instance).

Furthermore, in order to support distributed business process monitoring at the VE level, specific workflow plans are necessary to support real-time information gathering from the involved partners (implementation of remote federated query mechanisms). Figure 5 illustrates the complex interactions involving various PCL modules in order to feed the distributed business process management system 
(DBPMS) (Pereira Klen et al, 1998) of the VE coordinator with some requested information.

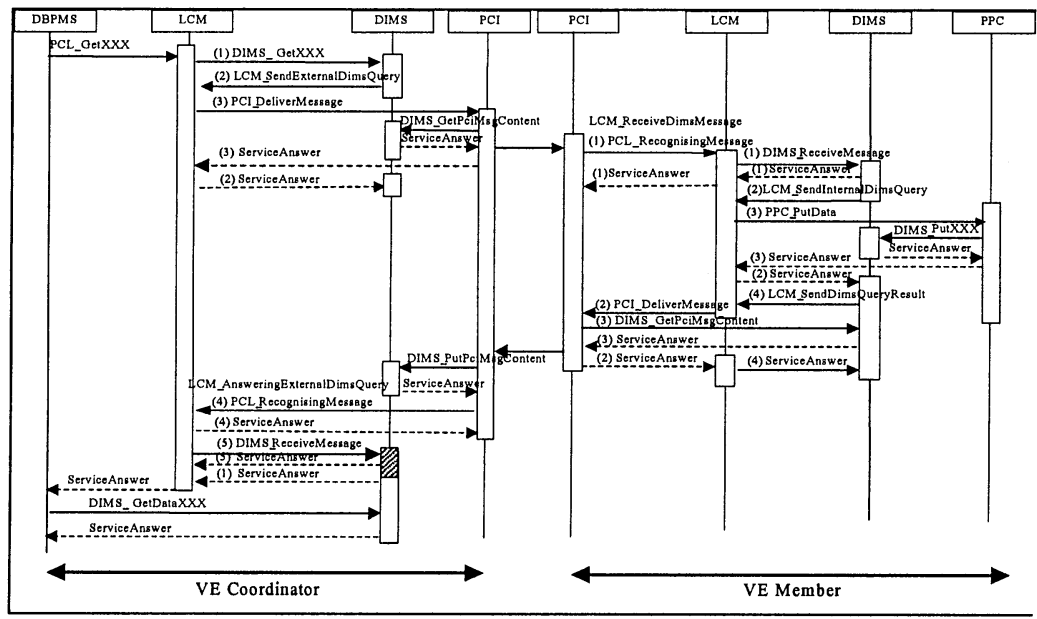

Figure 5 - Example of complex interactions among modules in the PCL

\section{CONCLUSIONS}

Configurable definition of coordination policies supported by a flexible coordination mechanism is a major issue in the design and development of a supporting infrastructure for virtual enterprises. In order to cope with these aspects, the infrastructure proposed by PRODNET adopted a hierarchical workflow-based coordination approach and a high degree of configurability, supporting both the preparation of the company to participate in a VE environment, the VE creation and (re-)configuration. Privacy and configurability of levels of information visibility necessary for information exchange as well as the support for distributed business process management are guaranteed by an innovative federated information management approach among VE-member enterprises.

Acknowledgements. This work was funded in part by the European Commission, Esprit program within the PRODNET II project. The authors also thank the valuable contributions from the consortium partners: CSIN (P), ESTEC (P), HERTEN (BR), Lichen Informatique (F), MIRALAGO (P), ProSTEP(D), Uninova (P), University of Amsterdam (NL), Universidade Federal de Santa Catarina (BR), and Universidade Nova de Lisboa (P). 


\section{REFERENCES}

Afsarmanesh, H; Camarinha, L. - Federated Information Management for Cooperative Information - Proc. of DEXA'97 - Int. Conf. on DB \& Expert Systems Applic., Sep 97.

Camarinha-Matos, L.M.; Afsarmanesh, H. - Infrastructures for Virtual Enterprises, Kluwer Academic Publishers (to appear), 1999.

Camarinha-Matos, L.M.; Afsarmanesh, H; Garita, C.; Lima, C. - Towards an architecture for virtual enterprises, J. of Intelligent Manufacturing, Vol. 9, Issue 2, Apr. 98, pp 189-199.

Pereira Klen, A. A.; Spinosa, L. M.; Rabelo, R.; Ferreira, A. - Integrated Logistics Management Support System: An Advanced Coordination Functionality for the Virtual Environment, Proc. of IMS'98 - $5^{\text {th }}$ IFAC Workshop on Intelligent Manufacturing Systems, Gramado, Brasil, 9-11 Nov 1998. 